\title{
Getting a sense of what is in your beverage
}

\author{
Shauna Scanlon
}

Tyndall National Institute and the Department of Chemistry , UCC

\section{Introduction}

When you drink a soft drink, probably the last thing that crosses your mind is how the taste is the exact same as the one you had before that, and the one before that. But how do the manufacturers ensure that the taste and quality of these beverages are consistent from one bottle to the next? Throughout the production process, food and beverage manufacturers must continuously ensure that their product meets the highest standards. In order to do this, a number of steps must be carried out. These begin with the analysts removing a sample of the product from the process line. Next, the product is taken from the processing plant and brought to the lab, where various quality control tests must be carried out. Here, it is the job of one or more analytical chemists to determine if the sample meets the standards expected by not only the quality control managers but also by the consumers who will buy the final product. The experiments carried out by the chemists include determining the $\mathrm{pH}$ of the sample, measuring the colour of the product, analysing the particle sizes within the sample, determining its viscosity and many more. Some of these experiments are quick, and simply involve placing a recording device into the sample and getting a reading. Others, however, are more complex and use very large instruments that may take almost an hour to provide a reading. The fact that the chemists must use each of these instruments repeatedly at regular intervals throughout the day, means that the current method of determining product quality is cumbersome, costly and laborious, where one cycle of testing may take up to two hours. Despite this, the analysis of product quality is a critical part of production and, therefore, manufacturers have had to accept these time-consuming methods as necessary steps to be carried out multiple times a day. What the food and beverage industry needs is new methods of measuring the quality of their products, which are easy to use, cost effective and use state-of-the-art technology.

\section{Outline of my research}

\section{Process Analytical Technology}

Consumers have increasingly high expectations when it comes to the quality of their food products and are looking for quality seals and trust marks on food and beverages, expecting manufacturers to produce these products to the highest standards. In order to 
achieve this, the implementation of Process Analytical Technology (PAT) in the food and beverage industry has become increasingly desirable. PAT can be described as a system for analysing, and controlling manufacturing through regular measurements of the critical parameters that contribute to the quality of in-process materials. This is done through the application of a variety of different analytical methodologies, such as the ones mentioned previously, either directly within the process line, or externally. In this way quality is built into the product rather than determined through end-product testing.

My research aims to develop a system which integrates all of the technologies required to carry out the necessary quality control experiments into a single device for use with PAT in the food and beverage industry. This system will consist of two separate devices. The first device will be used to detect temperature, $\mathrm{pH}$ and conductivity while the second device will be used to detect specific gravity, particle size and caffeine concentration.

So how can instruments of such a large size be integrated into a device measuring a few centimetres? The answer can be found in the emerging technological area of MicroElectro-Mechanical Systems, or MEMS.

\section{MEMS}

MEMS can simply be described as miniaturised devices and structures that are made using the technique of micro-fabrication, the most notable elements being microsensors. MEMS involve the integration of complete systems and technologies onto a single silicon chip whose dimensions can vary from a few micrometres to several millimetres. To give an indication of the size of this, the width of a single human hair ranges from 17 to $181 \mathrm{mi}-$ crometres. As the name suggests, MEMS are devices that consist of at least some elements that have mechanical functionality. This does not mean to say that the elements necessarily have to move and therefore MEMS can vary from relatively simple structures having no moving elements, to extremely complex electromechanical systems with multiple moving elements under the control of integrated microelectronics.

It is these microsensors which will form the main part of my research. By replacing the currently used analytical instruments with microsensors, a multi-parameter sensing system can be developed.

\section{Device components}

An example of a microsensor that will be integrated into the device is an Ion-Sensitive Field Effect Transistor (ISFET). ISFETs are sensors used to determine the $\mathrm{pH}$ of a substance. They differ from the traditionally used glass electrodes in that they do not have an external glass structure, which means that they are more robust. This is an advantage, especially in 


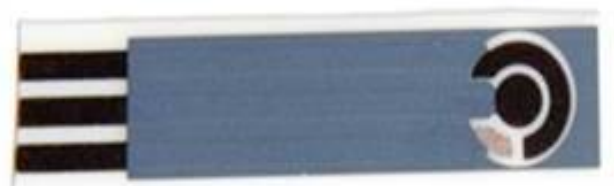

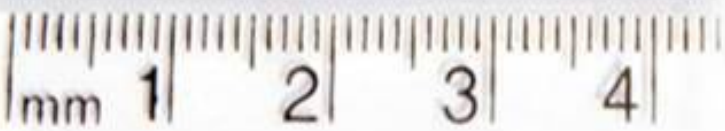 \\ $\mathrm{cm}$}

Figure 1: A Screen Printed Carbon Electrode for Caffeine detection. Image: Shauna Scanlon.

the food industry, where a glass breakage can bring the whole process to stop, no matter how small the glass fragments.

By using ISFETs instead of the currently used glass electrodes many other advantages will also be introduced such as their small size, rapid response and low cost.

Another example of a microsensor that will be used in the device is a conductivity sensor. This sensor gives an indication of, among other things, the consistency of the dissolved solids in the sample.

A planar sensor will be used to replace the traditional conductivity probe. An example of the dimensions of the sensor to be used, such as the one in Figure 3, is $15 \mathrm{~mm} \times 5.5 \mathrm{~mm}$ x $0.65 \mathrm{~mm}$, which is approximately half the size of a postage stamp.

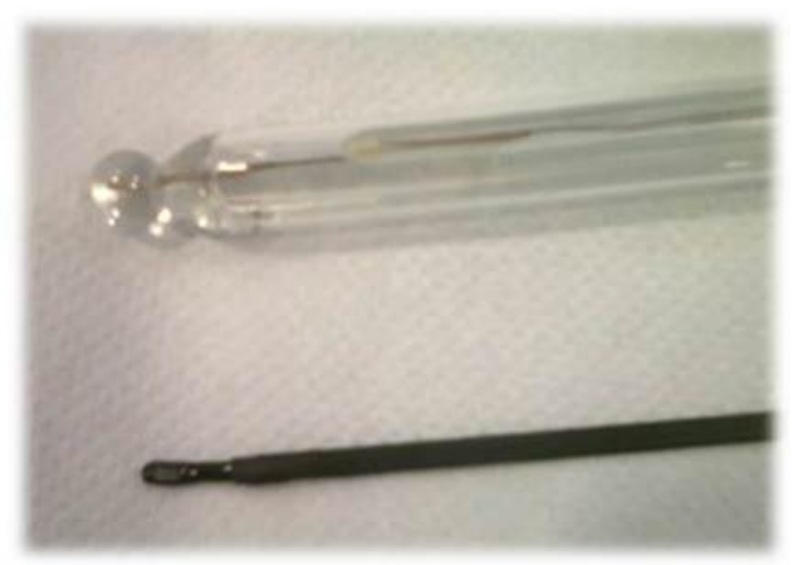

Figure 2: Comparison of a traditional pH glass electrode (above) and an ISFET $\mathrm{pH}$ probe with the ISFET sensor on the probe tip (below). Image: Shauna Scanlon. 

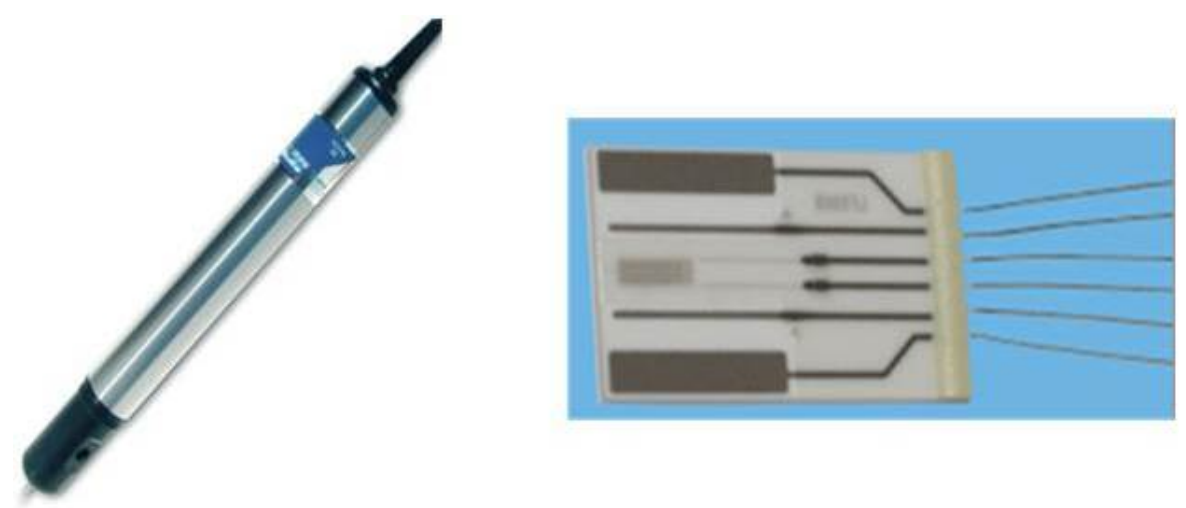

Figure 3: Traditional Conductivity probe (left), Planar, combined temperature and conductivity sensor (right). Image: IST AG.

\section{Integration of devices}

My multi-parameter sensing system will be integrated directly into the manufacturing process allowing for quality of the product to be continuously monitored, while avoiding disruption of manufacturing.

There are two methods by which the devices can be included in the manufacturing process. The first way is to fabricate a single probe that contains all of the sensors together on the probe tip. This could be integrated into the process line directly, with measurements being taken of the sample as it flows past during production.

The second method for integrating the devices would be through the use of an external sample cell that houses the sensors outside the process tank. In this case, small lines would be used to bring a sample from the main process line to the device, with another bringing the small amount of analysed sample to a waste area.

Either of these techniques enables all of the required tests of the sample to be carried out, while the quality of the product is continuously monitored. This will allow for manufacturers to receive the data they need, while avoiding the cost and time spent carrying out experiments in the laboratory.

Data from the sensors will be transmitted to the laboratory via a data aquisition system. This can then be analysed, trended and alarms points can be set up, which will be used to monitor any parameter that falls outside of the desired specification.

The approach to quality control, which I will be developing in my research, will open up further possibilities to exploit the technology in other relevant application sectors such as pharmaceutical and bioprocessing industries.

So, next time you have a soft drink, you can be happy in the knowledge that manufacturers are using state-of-the-art technology to assure the highest standards of taste and quality for you. 
I would like to thank Dr Eric Moore, my supervisor, in Tyndall National Institute and Dr Damien Browne and Dr Sharon Rothwell, my co-supervisors, in PepsiCo for their support and advice. I would also like to acknowledge the Irish Research Council and PepsiCo for funding my research through the Enterprise Partnership Scheme. 